\title{
Traumatic aniridia
}

\author{
M. ROMEM AND L. SINGER \\ Department of Ophthalmology, Government Hospital, Yafo, Tel Aviv University Medical School, \\ Israel
}

Traumatic aniridia or irideremia occurs mostly after a severe blow on the eyeball and is usually accompanied by its rupture. Less frequently it is seen after a perforating wound. Traumatic aniridia is invariably followed by a complete hyphaema, and very frequently by severe disorganization of the ocular tissue causing massive vitreous haemorrhage. The aniridia is, as a rule, discovered only after absorption of the blood from the anterior chamber, and the aspect of a completely black eye instead of one with a pupil is remarkable.

The torn-off iris may be retracted as a grey globule in the anterior chamber and may obstruct the angle. It may escape through the wound and disappear or be dislocated under the conjunctiva. The literature was expertly summarized by Duke-Elder (1954).

\section{Case report}

A 56-year-old man was struck on the left eye by a wooden plank, which caused a perforating corneo-scleral wound extending temporally from the limbus down to the equator. A vitreous bead was incarcerated in the wound and the anterior chamber and vitreous cavity were filled with blood.

Multiple glass fragments from the patient's broken spectacles were lying on the cornea and in the lower fornix; these were removed and the wound was repaired.

It was discovered 2 weeks later that there was no iris present at all. The visual acuity was reduced to perception of light since the vitreous was still full of blood. The lens was in place and all the zonular fibres were intact. The intraocular pressure was high in spite of intensive antiglaucomatous treatment.

After 2 months the visual acuity had improved to finger counting at $4 \mathrm{~m}$. Gonioscopy showed that the angle of the anterior chamber was obstructed by a layer of clotted blood, and tonographic measurements showed a coefficient of outflow of $0 \cdot 08$.

After a further 9 months, the visual acuity had improved to $6 / 6$ with-I.5 D sph. through a stenopoeic hole. No trace of the iris could be detected in the anterior chamber, the vitreous cavity, or under the conjunctiva. By this time all the blood had absorbed and the coefficient of outflow had increased to $0 \cdot 20$. The trabeculae looked normal and no signs of a recessed angle were present. The intraocular pressure could be lowered to about $25 \mathrm{~mm}$. $\mathrm{Hg}$ by the use of pilocarpine, Epitrate, and Diamox.

The visual acuity of $6 / 6$ and all other findings have remained unchanged during a follow-up period of 2 years. Every attempt to reduce the antiglaucomatous treatment was followed by a rise in tension, but operative intervention has been put off for the time being. A coloured contact lens has been prescribed for the aniridic eye to reduce the photophobia and dazzling that have resulted from the absence of the iris.

The patient's right eye is completely normal and the visual acuity $6 / 6$ with-I $\cdot 5 \mathrm{D}$ sph. All provocative tests for glaucoma in this eye were negative.

\section{Comment}

The restoration of normal vision and the general recovery of the eye after an injury so severe as to lead to complete aniridia, although not unique, is surprising. 
It is not known how the iris managed to escape without any trace from the anteriog chamber. Since the zonular fibres were found to be intact, the only available escape route was the short distance of the incision which extended into the cornea at the limbus $\overrightarrow{\vec{m}}$ The glass splinter which had cut the wound must have engaged the prolapsed iris at this point and have torn it out, the impinging wooden plank being only the indirect caus of the injury.

The therapy-resistant glaucoma which followed the injury is reminiscent of the glaucomå seen in congenital aniridia, though in our case no fragments of iris remained to occlude the्e angle. Traumatized trabeculae cannot be excluded as the cause of high tension although $\vec{p}$ gonioscopically they looked normal. The assumption that the absence of an aqueous $\vec{\omega}$ resorbing surface is the cause of the hypertension remains conjectural.

\title{
Summary
}

A case is described of traumatic aniridia due to the impact of a wooden plank and $\stackrel{\circ}{\mathrm{a}}$ perforating corneo-scleral wound caused by broken spectacles. In spite of the severe injury, the eye recovered normal vision. The mechanism of the uprooting of the iris and its escape out of the eye, and that of the persistent secondary glaucoma, are discussed

\section{Reference}

DUKE-ELDER, s. (1954) “Text-book of Ophthalmology”, vol. 6, pp. 5789 and 6or6. Kimpton; London

\section{Rupture of the globe by a snail-shell}

\author{
F. A. HOSNI \\ Qatar, Arabian Gulf
}

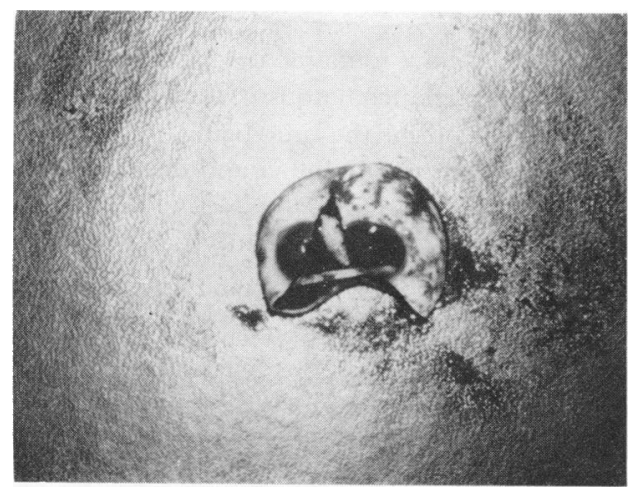

An 8-year-old boy was playing with an? old gun into which he had put a snail ț shell. The gun was suddenly fired and the shell was projected into the eyeball Radiography showed the snail-shell withing the orbital cavity. Unfortunately, the eyeball had to be excised as it was badly damaged with complete loss of vision? The cut section of the globe (Figure) shows the shell within the vitreous cavity

FIGURE Section of globe, showing snail-shell within the vitreous cavity. 